\title{
Homogeneous Subspaces of Products of Extremally Disconnected Spaces
}

\author{
Evgenii Reznichenko*
}

\section{Introduction}

In [1] it was proved that the product of pseudocompact groups is pseudocompact. In [2] the compactness-type properties of products of homogeneous spaces were studied. In particular, homogeneous pseudocompact spaces $X$ and $Y$ whose product $X \times Y$ is not pseudocompact was constructed [2, Theorem 1.1]. Under Martin's axiom homogeneous countably compact spaces $X$ and $Y$ whose product $X \times Y$ is not pseudocompact were also constructed [2, Theorem 4.1] and the problem of the existence of such spaces without additional set-theoretic assumptions was posed [2, Question 5.1(a)]) . In this paper we construct such an example (Theorem 1). However, the problem of the existence of a homogeneous pseudocompact space $X$ for which the product $X \times X$ is not pseudocompact [2, Question 5.3] still remains open.

In [3] products of homogeneous extremally disconnected spaces were studied. Under Martin's axiom homogeneous extremally disconnected countably compact spaces $X$ and $Y$ with non-countably compact product $X \times Y$ were constructed [3, Theorem 4.2]. In [5] this example was improved; namely, the product $X \times Y$ was made nonpseudocompact [5, Theorem 3]. In [4 homogeneous extremally disconnected countably compact spaces $X$ and $Y$ for which the product $X \times$ $Y$ is not countably compact were constructed without additional set-theoretic assumptions [4, Theorem 4.1].

The homogeneous extremally disconnected countably compact spaces constructed in [3, 4, 5] are maximally homogeneous (see Definition 4 below). In this paper we prove that the countable power of a maximally homogeneous extremally disconnected countably compact space is countably compact (Corollary $[3]$.

In [8] Arhangel'skii proved that any compact subset of an extremally disconnected topological group is finite. At the same time, Frolíc proved that homogeneous extremally disconnected compact spaces are finite [6]. In this paper we obtain a simultaneous generalization of these two theorems; namely, we prove that all compact subsets of homogeneous extremally disconnected spaces are finite (Corollary 4), which answers Questions 4.5.2 and 4.5.3 in [7]. This

*Supported by RFBR, project no. 17-51-18051 Bolg_a 
statement follows also from Theorem 2(c) of [22]. It can be strengthened: all compact subsets of homogeneous subspaces of the third power of an extremally disconnected space are finite (Theorem 5). The author is unaware of whether this is true for the fourth power without additional set-theoretic assumptions (Question 2). However, under $\mathrm{CH}$, it is true. Moreover, under $\mathrm{CH}$, all compact subsets of homogeneous subspaces of any finite power of an extremally disconnected space are finite (Theorem 4) and all compact subsets of homogeneous subspaces of the countable power of an extremally disconnected space are metrizable (Theorem 3). It is unknown whether the last statement can be proved naïvely (Question 1).

We also strengthen Frolík's theorem mentioned above by proving that all compact homogeneous subspaces of finite powers of an extremally disconnected space are finite (Theorem [6).

\section{Conventions, Definitions, and Notation}

All spaces considered in this paper are assumed to be completely regular and Hausdorff. The set of positive integers is denoted by $\omega, \beta \omega$ is the set of all ultrafilters on $\omega$, and $\omega^{*}=\beta \omega \backslash \omega$ is the set of free ultrafilters on $\omega$. Given a space $X$ and a set $M \subset X$, by $\bar{M}^{X}$ and $\operatorname{int}_{X}(M)$ we denote, respectively, the closure and the interior of $M$ in $X$. When it is clear from the context what ambient space $X$ is meant, we write simply $\bar{M}$ and $\operatorname{int}(M)$ instead of $\bar{M}^{X}$ and $\operatorname{int}_{X}(M)$. By id $\operatorname{lo~}_{X}$ we denote the identity self-map of $X$.

Recall that a space $X$ is said to be extremally disconnected $(E D)$ if $\bar{U} \cap \bar{V}=\varnothing$ for any disjoint open sets $U, V \subset X$. A completely regular Hausdorff space is called an $F$-space if every cozero set is $C^{*}$-embedded in this space. Elementary properties of F-spaces and ED spaces can be found in the book 12 by Gillman and Jerison. Any ED space is an F-space.

In a space $X$ is called a $\beta \omega$ space if, given any countable discrete set $M \subset X$ with compact closure $\bar{M}$, its closure is homeomorphic to $\beta \omega$ [15]. Any $F$-space is a $\beta \omega$ space, and any subspace of a $\beta \omega$ space is a $\beta \omega$ space.

Let us remind the definition of the Keisler-Rudin order $\leq_{K R}$ on $\omega^{*}$. Let $p, q \in \omega^{*}$. Then $p \leq_{K R} q$ if and only if there exists a map $f: \omega \rightarrow \omega$ such that $\beta f(q)=p$. Here $\beta f: \beta \omega \rightarrow \beta \omega$ is the continuous extension of the map $f$.

Let $X$ be space. We say that a sequence $\zeta=\left(x_{n}\right)_{n \in \omega}$ on $X$ is exact if $x_{n} \neq x_{m}$ for $n \neq m$ and discrete if the set $\left\{x_{n}: n \in \omega\right\}$ is discrete.

We use the following notation:

- $\mathfrak{S}(X)=X^{\omega}$ is the set of all sequences on $X$;

- $\mathfrak{S}_{k}(X)$ is the set of sequences on $X$ whose ranges have compact closure in $X$;

- $\mathfrak{S}_{d}(X)$ is the set of discrete exact sequences on $X$;

- $\mathfrak{S}_{d k}(X)=\mathfrak{S}_{d}(X) \cap \mathfrak{S}_{k}(X)$ is the set of discrete exact sequences on $X$ whose ranges have compact closure. 
Let $\mathcal{T}$ denote the topology of $X$, and let $\mathcal{T}_{*}=\mathcal{T} \backslash\{\varnothing\}$. Then

- $\mathfrak{O}(X)=\mathcal{T}_{*}^{\omega}$ is the set of sequences of nonempty open subsets of $X$;

- $\mathfrak{O}_{d}(X)$ is the set of disjoint sequences of nonempty open subsets of $X$.

Given a sequence $\xi=\left(M_{n}\right)_{n \in \omega}$ of subsets of $X$, a point $x \in X$, and a set $M \subset X$, we put

$$
\begin{aligned}
u_{x}(\xi)=\left\{p \in \omega^{*}:\left\{n \in \omega: U \cap M_{n} \neq \varnothing\right\} \in p\right. \\
\\
\text { for each neighborhood } U \text { of } x\} ;
\end{aligned}
$$

- $u_{M}(\xi)=\bigcup_{x \in M} u_{x}(\xi)$;

- $u(\xi)=u_{X}(\xi)$.

For a sequence $\zeta=\left(x_{n}\right)_{n \in \omega}$ of points on $X$, we set

\section{•}

$$
\begin{aligned}
u_{x}(\zeta)=\left\{p \in \omega^{*}:\left\{n \in \omega: x_{n} \in U\right\} \in p\right. \\
\text { for each neighborhood } U \text { of } x\} ;
\end{aligned}
$$

- $u_{M}(\zeta)=\bigcup_{x \in M} u_{x}(\zeta)$;

- $u(\zeta)=u_{X}(\zeta)$.

Note that, for $\zeta^{\prime}=\left(\left\{x_{n}\right\}\right)_{n \in \omega}$, we have $u_{x}(\zeta)=u_{x}\left(\zeta^{\prime}\right), u_{M}(\zeta)=u_{M}\left(\zeta^{\prime}\right)$, and $u(\zeta)=u\left(\zeta^{\prime}\right)$.

We also set

- $\operatorname{sp}(x, X)=\bigcup_{\zeta \in \mathfrak{S}_{d}(X)} u_{x}(\zeta)$;

- $\operatorname{sp}(X)=\bigcup_{x \in X} \operatorname{sp}(x, X)$;

- $\operatorname{sp}_{k}(X)=\bigcup_{\zeta \in \mathfrak{S}_{d k}(X)} u_{x}(\zeta)$;

- $\operatorname{sp}_{k}(X)=\bigcup_{x \in X} \operatorname{sp}_{k}(x, X)$.

Note that $p \in u_{x}(\zeta)$ if and only if $x$ is the $p$-limit of the sequence $\zeta$. We denote the $p$-limit of $\zeta$ by $\lim _{p} \zeta$. 


\section{Sequences on Spaces}

Definition 1. We say that a sequence $\zeta=\left(x_{n}\right)_{n \in \omega}$ is almost exact if there exists an $N \in \omega$ such that $x_{n} \neq x_{m}$ for $n \neq m$ and $n, m>N$. We say that $\zeta$ is almost stationary if there exists an $N \in \omega$ such that $x_{n}=x_{m}$ for $n, m>N$.

Statement 1. Suppose that $X=\prod_{n \in \omega} X_{n}$ is a countable product of spaces, $M \subset X$ is an infinite subset of $X$, and $\pi_{n}: X \rightarrow X_{n}$ is the projection for each $n \in \omega$. Then there exists an exact sequence $\left(x_{k}\right)_{k \in \omega} \subset M$ such that $\left(\pi_{n}\left(x_{k}\right)_{k \in \omega}\right.$ is an almost stationary or almost exact discrete sequence in $X_{n}$ for each $n$.

Proof. It is easy to construct, by induction, a sequence of infinite sets $\left(M_{n} \subset\right.$ $M)_{n \in \omega}$ such that, for each $n, M_{n+1} \subset M_{n}$ and either $\left|\pi_{n}\left(M_{n}\right)\right|=1$ or $\pi_{n}\left\lceil_{M}\right.$ is an injective map and the set $\pi_{n}\left(M_{n}\right)$ is discrete in $X_{n}$. It remains to take an exact sequence $\left(x_{k}\right)_{k \in \omega}$ such that $x_{k} \in M_{k}$ for all $k$.

Statement 2. Let $X$ be a space, and let $\left(U_{n}\right)_{n \in \omega} \in \mathfrak{O}(X)$. Then there exists an infinite set $M \subset \omega$ and a family of nonempty open sets $\left(V_{n}\right)_{n \in M}$ such that $V_{n} \subset U_{n}$ for $n \in M$ and one of the following conditions holds:

(1) the family $\left\{V_{n}: n \in M\right\}$ is disjoint;

(2) all $V_{n}$ coincide and consist of one point.

Proof. Consider two cases.

The first case. For any finite set $K$, the complement $U_{n} \backslash K$ is nonempty for infinitely many $n$. Then it is easy to inductively construct an infinite set $M^{\prime} \subset \omega$ and a sequence $\left(x_{n}\right)_{n \in M^{\prime}}$ such that $x_{n} \in U_{n}$ and $x_{m} \neq x_{n}$ for any different $n, m \in M^{\prime}$. Choose an infinite set $M \subset M^{\prime}$ so that $\left\{x_{n}: n \in M\right\}$ is discrete. Take neighborhoods $W_{n}$ of $x_{n}$ for which the family $\left\{W_{n}: n \in M\right\}$ is disjoint. The family of $V_{n}=U_{n} \cap W_{n}$ satisfies condition (1).

The second case. There exists a finite set $K \subset X$ which contains almost all $U_{n}$. In this case, we can find its subset $U \subset K$ and an infinite set $M \subset \omega$ such that $U_{n}=U$ for $n \in M$. Choose $u \in U$. The family of $V_{n}=\{u\}, n \in M$, satisfies condition (2).

Statement 3. Let $X=\prod_{n \in \omega} X_{n}$ be a countable product of spaces, and let $\left(W_{k}\right)_{k \in \omega} \in \mathfrak{O}(X)$. Then there exists an increasing sequence $\left(n_{k}\right)_{k \in \omega} \subset \omega$ and sequences $\left(U_{n, k}\right)_{k \in \omega} \in \mathfrak{O}\left(X_{n}\right), n \in \omega$, such that $\prod_{n \in \omega} U_{n, k} \subset W_{n_{k}}$ and, for each $n \in \omega$, the family $\zeta_{n}=\left\{U_{n, k}: k>n\right\}$ satisfies one of the following conditions:

(1) $\zeta_{n}$ is disjoint;

(2) $\zeta_{n}$ consists of coinciding one-point sets.

Proof. There exist $\left(V_{n, k}\right)_{k \in \omega} \in \mathfrak{O}\left(X_{n}\right), n \in \omega$, such that $\prod_{n \in \omega} V_{n, k} \subset W_{k}$.

Using Statement 2, we can easily construct a decreasing sequence $\left(M_{n}\right)_{n \in \omega}$ infinite subsets of $\omega$ and a family of sequences $\left(Q_{n, k}\right)_{k \in \omega} \in \mathfrak{O}\left(X_{n}\right)$ so that $Q_{n, k} \subset V_{n, k}$ for all $n, k$ and one of the following conditions holds: 
(1) $\left\{Q_{n, k}: k \in M_{n}\right\}$ is disjoint;

(2) $\left\{Q_{n, k}: k \in M_{n}\right\}$ consists of coinciding one-point sets.

It remains to take an increasing sequence $\left(n_{k}\right)_{k \in \omega} \subset \omega$ with $n_{k} \in M_{k}$ for $k \in \omega$ and put $U_{n, k}=Q_{n, n_{k}}$ for $k, n \in \omega$.

Statement 4. For any space $X$ and any sequences $\left(U_{n}\right)_{n \in \omega},\left(V_{n}\right)_{n \in \omega} \in \mathfrak{O}_{d}(X)$, one of the following conditions holds:

(1) $U_{n}=V_{n}$ and $\left|U_{n}\right|=1$ for almost all $n$;

(2) there exists an infinite set $M \subset \omega$ and families $\left(U_{n}^{\prime}\right)_{n \in M}$ and $\left(V_{n}^{\prime}\right)_{n \in M}$ of nonempty open sets such that $\bigcup_{n \in M} U_{n}^{\prime} \cap \bigcup_{n \in M} V_{n}^{\prime}=\varnothing$ and $U_{n}^{\prime} \subset U_{n}$, $V_{n}^{\prime} \subset V_{n}$ for all $n \in M$.

Proof. Suppose that (1) does not hold. Then there exists an infinite set $M^{\prime} \subset \omega$ and families $\left(U_{n}^{\prime \prime}\right)_{n \in M}$ and $\left(V_{n}^{\prime \prime}\right)_{n \in M}$ of nonempty open sets such that $U_{n}^{\prime \prime} \cap V_{n}^{\prime \prime}=$ $\varnothing$ and $U_{n}^{\prime \prime} \subset U_{n}, V_{n}^{\prime \prime} \subset V_{n}$ for all $n \in M^{\prime}$. We set $A_{n}=\left\{m \in M^{\prime}: U_{n}^{\prime \prime} \cap V_{m}^{\prime \prime} \neq\right.$ $\varnothing, m>n\}$ and $B_{n}=\left\{m \in M^{\prime}: U_{m}^{\prime \prime} \cap V_{n}^{\prime \prime} \neq \varnothing, m>n\right\}$ for $n \in M^{\prime}$. Consider three cases.

The first case. The set $A_{n}$ is infinite for some $n \in M^{\prime}$. In this case, we take $M=A_{n}$ and put $U_{m}^{\prime}=U_{m}^{\prime \prime}$ and $V_{m}^{\prime}=V_{m}^{\prime \prime} \cap U_{n}$ for $m \in M$.

The second case. The set $B_{n}$ is infinite for some $n \in M^{\prime}$. In this case, we take $M=B_{n}$ and put $U_{m}^{\prime}=U_{m}^{\prime \prime} \cap V_{n}$ and $V_{m}^{\prime}=V_{m}^{\prime \prime}$ for $m \in M$.

The third case. The sets $A_{n}$ and $B_{n}$ are finite for all $n \in M^{\prime}$. In this case, there exists a sequence $\left(m_{k}\right)_{k \in \omega} \subset M^{\prime}$ such that $m_{k+1}>m_{k}, m_{k+1}>\max A_{m_{k}}$, and $m_{k+1}>\max B_{m_{k}}$ for all $k$. We take $M=\left\{m_{k}: k \in \omega\right\}$ and put $U_{m}^{\prime}=U_{m}^{\prime \prime}$ and $V_{m}^{\prime}=V_{m}^{\prime \prime} \cap U_{n}$ for $m \in M$.

\section{Extremally Disconnected Spaces and Ultrafilters}

Statement 5 ([12, 13, 14]). The following assertions are equivalent for any completely regular Hausdorff space $X$ :

(1) $X$ is $E D$;

(2) every dense subset of $X$ is $E D$;

(3) every open subset of $X$ is $E D$;

(4) every dense subset of $X$ is $C^{*}$-embedded;

(5) every open subset of $X$ is $C^{*}$-embedded.

The following assertion is a direct consequence of definitions.

Statement 6. A space $X$ is a $\beta \omega$ space if and only if $\left|u_{x}(\zeta)\right| \leq 1$ for any $x \in X$ and $\zeta \in \mathfrak{S}_{d k}(X)$. 
Proposition 1. Let $X$ be a space.

(1) If $X$ contains an infinite compact set, then $\operatorname{sp}_{k}(X)=\omega^{*}$.

(2) If $X$ is homogeneous, then $\operatorname{sp}(X)=\operatorname{sp}(x, X)$ and $\operatorname{sp}_{k}(X)=\operatorname{sp}_{k}(x, X)$ for any $x \in X$.

Proof. (1) Since $X$ contains an infinite compact set, it follows that $\mathfrak{S}_{d k}(X) \neq \varnothing$. If $\zeta \in \mathfrak{S}_{d k}(X)$, then $u_{X}(\zeta)=\omega^{*}$.

(2) Since $X$ is homogeneous, it follows that $\operatorname{sp}(x, X)=\operatorname{sp}(y, X)$ and $\operatorname{sp}_{k}(x, X)=\operatorname{sp}_{k}(y, X)$ for $x, y \in X$.

Proposition 2 (Frolík, [6]). Let $X$ be an ED space, and let $x \in X$. Then the set $\operatorname{sp}(x, X)$ is totally ordered with respect to the Keisler-Rudin order.

Proposition 3. Let $X$ be a $\beta \omega$ space, and let $x \in X$. Then the set $\operatorname{sp}_{k}(x, X)$ is totally ordered with respect to the Keisler-Rudin order.

Proof. Let $p, q \in \operatorname{sp}_{k}(x, X)$. Then we have $\{p\}=u_{x}(\zeta)$ and $\{q\}=u_{x}(\zeta)$ for some $\zeta, \xi \in \mathfrak{S}_{d k}(X)$. Let $M$ be the set of isolated points in $\zeta \cup \xi$, and let $K=\bar{M}$. Then $\zeta, \xi \subset K$ and $p, q \in \operatorname{sp}(x, K)$. Since $K$ is homeomorphic to $\beta \omega$ and extremally disconnected, it follows by Proposition 2 that $p$ and $q$ are $\leq_{K R}$-comparable.

Proposition 4 (Kunen, [16, Lemma 4]). Let $p, q \in \omega^{*}$ be $\leq_{K R}$-incomparable weak P-points, and let $X$ be any compact F-space. Suppose that $\zeta=\left(x_{n}\right)_{n \in \omega} \in$ $\mathfrak{S}_{d}(X), \xi=\left(y_{n}\right)_{n \in \omega} \in \mathfrak{S}(X)$, and $x=\lim _{p} \zeta=\lim _{q} \xi$. Then $\left\{n: y_{n}=x\right\} \in q$.

Proposition 4 implies the following assertion.

Proposition 5. Let $p, q \in \omega^{*}$ be $\leq_{K R}$-incomparable weak P-points, and let $X$ be any F-space. Suppose that $\zeta=\left(x_{n}\right)_{n \in \omega} \in \mathfrak{S}_{d k}(X), \xi=\left(y_{n}\right)_{n \in \omega} \in \mathfrak{S}_{k}(X)$, and $x=\lim _{p} \zeta=\lim _{q} \xi$. Then $\left\{n: y_{n}=x\right\} \in q$.

Proposition 6 (Simon, [19; see also [18, 17]). There exists a set $C \subset \omega^{*}$, $|C|=2^{2^{\omega}}$, consisting of pairwise $\leq_{K R}$-incomparable weak P-ultrafilters.

Proposition 7 (20]). (CH) There exists a set $C \subset \omega^{*},|C|=2^{2^{\omega}}$, consisting of pairwise $\leq_{K R}$-incomparable selective ultrafilters.

Statement 7. Suppose that $X$ is a $\beta \omega$ space, $A$ is a set, $K$ is a compact subspace of $X^{A}$, and one of the following conditions holds:

(1) $A$ is finite and $K$ is infinite;

(2) $A$ is infinite and $w(K)>|A|$.

Then $\beta \omega$ is embedded in $K$.

Proof. For each $\alpha \in A$, let $\pi_{\alpha}: X^{A} \rightarrow X$ denote the projection onto the $\alpha$ th factor. There exists an $\alpha \in A$ for which $\pi_{\alpha}(K)$ is infinite. Let $M$ be a countable discrete subspace of $K$ for which $\pi_{\alpha} \uparrow_{M}$ is injective and $\pi_{\alpha}(M)$ is a discrete subspace of $X$. Then $\bar{M}$ is homeomorphic to $\beta \omega$. 
Statement 8. Suppose that $X$ is an $\beta \omega$ space, $p \in \omega^{*}$ is a selective ultrafilter, $\zeta=\left(z_{n}\right)_{n \in \omega} \in \mathfrak{S}\left(X^{\omega}\right)$, and $z=\lim _{p} \zeta \in X^{\omega}$. Suppose also that $\{z\} \cup\left\{z_{n}: n \in\right.$ $M\}$ is nonmetrizable for any $M \in p$. Let $\pi_{k}: X^{\omega} \rightarrow X$ denote the projection of $X^{\omega}$ onto the $k$ th factor. Then there exists an $m \in \omega$ and an $N \in p$ such that the sequence $\left(\pi_{m}\left(z_{n}\right)\right)_{n \in N}$ is discrete and exact, i.e., the set $\left\{\pi_{m}\left(z_{n}\right): n \in N\right\}$ is discrete and $\pi_{m}\left(x_{j}\right) \neq \pi_{m}\left(x_{i}\right)$ for any different $i, j \in N$.

Proof. First, we take $m \in \omega$ such that $\pi_{m}(M)$ is infinite for each $M \in p$. Such an $m$ exists. Indeed, otherwise, we can choose $M_{m} \in p$ so that $\left|\pi_{m}\left(M_{m}\right)\right|=1$ for each $m \in \omega$. Since the ultrafilter $p$ is selective, there exists an $M \in p$ such that $M_{m} \backslash M$ is finite for each $m \in \omega$. The set $\{z\} \cup\left\{z_{n}: n \in M\right\}$ is metrizable, which contradicts the assumption.

Let $\left(U_{n}\right)_{n \in \omega}$ be a sequence of neighborhoods of the point $\pi_{m}(z)$ for which $\pi_{m}(\zeta) \cap \bigcap_{n \in \omega} U_{n}$ contains at most one point and $U_{i+1} \subset \overline{U_{i+1}} \subset U_{i}$ for $i \in \omega$. Let $\left.N_{n}=\left\{i \in \omega: \pi_{m}(x) i\right) \in U_{n}\right\}$. Then $N_{n} \in p$. Since the ultrafilter $p$ is selective, we can find $N \in p$ such that $\left|N \cap N_{i} \backslash N_{i+1}\right| \leq 1$ for $i \in \omega$. The set $\left\{\pi_{m}\left(z_{n}\right): n \in N\right\}$ is discrete, and $\pi_{m}\left(x_{j}\right) \neq \pi_{m}\left(x_{i}\right)$ for different $i, j \in N$.

\section{Countably Compact and Pseudocompact Product Spaces}

The following assertion is easy to verify.

Statement 9. Suppose that $\left\{X_{\alpha}: \alpha \in A\right\}$ is a family spaces, $\zeta_{\alpha}=\left(R_{\alpha, n}\right)_{n \in \omega}$ is a sequence of nonempty subsets of $X_{\alpha}$ for each $\alpha$, and $R_{n}=\prod_{\alpha \in \omega} R_{\alpha, n}$. Then the sequence $\left.\left(R_{n}\right)\right)_{n \in \omega}$ is locally finite (or, in other words, has no accumulation points) in $\prod_{\alpha \in A} X_{\alpha}$ if and only if $\bigcap_{\alpha \in A} u_{X_{\alpha}}\left(\zeta_{\alpha}\right)=\varnothing$.

Proposition 8. Let $X$ be a space, and let $\tau$ be a cardinal.

(a) The following conditions are equivalent:

(1) $X$ is countably compact;

(2) $u(\zeta) \neq \varnothing$ for all $\zeta \in \mathfrak{S}(X)$;

(3) $u(\zeta) \neq \varnothing$ for all $\zeta \in \mathfrak{S}_{d}(X)$.

(b) The following conditions are equivalent:

(1) $X^{\omega}$ is countably compact;

(2) $\bigcap_{\zeta \in \gamma} u(\zeta) \neq \varnothing$ for all $\gamma \subset \mathfrak{S}(X)$ with $|\gamma| \leq \omega$;

(3) $\bigcap_{\zeta \in \gamma} u(\zeta) \neq \varnothing$ for all $\gamma \subset \mathfrak{S}_{d}(X)$ with $|\gamma| \leq \omega$.

(c) The following conditions are equivalent:

(1) $X^{\tau}$ is countably compact;

(2) $\bigcap_{\zeta \in \gamma} u(\zeta) \neq \varnothing$ for all $\gamma \subset \mathfrak{S}(X)$ with $|\gamma| \leq \tau$. 
Proof. Statement 9 implies the equivalence of conditions (1) and (2) in assertions (a), (b), and (c). The implications (2) $\Longrightarrow(3)$ in (a) and (b) are obvious. The implication $(3) \Longrightarrow(2)$ in (a) follows from the fact that each countable set contains a discrete countable subset.

Let us prove that $(3) \Longrightarrow(2)$ in (b). Let $\pi_{n}$ denote the projection of $X^{\omega}$ onto the $n$th factor. Take an infinite set $M \subset X$. Statement 1 implies the existence of an exact sequence $\left(x_{k}\right)_{k \in \omega} \subset M$ such that $\left(\pi_{n}\left(x_{k}\right)_{k \in \omega}\right.$ is an almost stationary or almost exact discrete sequence in $X$. In view of (3), we have $P=\bigcap_{\zeta \in \gamma} u(\zeta) \neq \varnothing$, where $\gamma=\left\{\left(\pi_{n}\left(x_{k}\right)_{k \in \omega}: n \in \omega\right\}\right.$. Take $p \in P$. Let $y_{n}$ be the $p$-limit of the sequence $\pi_{n}\left(x_{k}\right)_{k \in \omega}$, and let $y=\left(y_{n}\right)_{n \in \omega}$. Then the point $y$ is the $p$-limit of $\left(x_{k}\right)_{k \in \omega}$. Therefore, $y$ is an accumulation point for the set $M$.

Proposition 9. Let $X$ be a space, and let $\tau$ be an infinite cardinal.

(a) The following conditions are equivalent:

(1) $X$ is pseudocompact;

(2) $u(\zeta) \neq \varnothing$ for all $\zeta \in \mathfrak{O}(X)$;

(3) $u(\zeta) \neq \varnothing$ for all $\zeta \in \mathfrak{O}_{d}(X)$.

(b) The following conditions are equivalent:

(1) $X^{\omega}$ is pseudocompact;

(2) $\bigcap_{\zeta \in \gamma} u(\zeta) \neq \varnothing$ for all $\gamma \subset \mathfrak{O}(X)$ with $|\gamma| \leq \omega$;

(3) $\bigcap_{\zeta \in \gamma} u(\zeta) \neq \varnothing$ for all $\gamma \subset \mathfrak{O}_{d}(X)$ with $|\gamma| \leq \omega$;

(4) $X^{\tau}$ is pseudocompact.

Proof. Assertion (a) follows from Statement 2, Let us prove (b).

$(1) \Longrightarrow(2)$ Let $\gamma=\left\{\left(U_{n, k}\right)_{k \in \omega}: n \in \omega\right\} \subset \mathfrak{O}(X)$. We set

$$
V_{n, k}= \begin{cases}X, & k<n \\ U_{n, k}, & k \geq n\end{cases}
$$

Note that $u\left(\left(U_{n, k}\right)_{k \in \omega}\right)=u\left(\left(V_{n, k}\right)_{k \in \omega}\right)$. The set $V_{k}=\prod_{n \in \omega} V_{n, k}$ is open in $X^{\omega}$. It follows from the pseudocompactness of $X^{\omega}$ that the family $\left(V_{k}\right)_{k \in \omega}$ is not locally finite. Statement 9 implies $\bigcap_{\zeta \in \gamma} u(\zeta)=\bigcap_{n \in \omega} u\left(\left(V_{n, k}\right)_{k \in \omega}\right) \neq \varnothing$.

$(2) \Longrightarrow(1)$. Let $\left(W_{k}\right)_{k \in \omega} \in \mathfrak{O}\left(X^{\omega}\right)$. There exist sequences $\left(V_{n, k}\right)_{k \in \omega} \in$ $\mathfrak{O}(X)$ such that $\prod_{n \in \omega} V_{n, k} \subset V_{k}$. By virtue of Statement 9 implies that $\left(V_{k}\right)_{k \in \omega}$ is not locally finite.

The implication $(2) \Longrightarrow(3)$ is obvious.

$(3) \Longrightarrow(1)$. Let $\left(W_{k}\right)_{k \in \omega} \in \mathfrak{O}\left(X^{\omega}\right)$. By virtue of Statement 3 there exists an increasing sequence $\left(n_{k}\right)_{k \in \omega} \subset \omega$ and sequences $\left(U_{n, k}\right)_{k \in \omega} \in \mathfrak{O}(X), n \in \omega$, such that $\prod_{n \in \omega} U_{n, k} \subset W_{n_{k}}$ and, for each $n \in \omega$, the family $\zeta_{n}=\left\{U_{n, k}: k>n\right\}$ satisfies one of the following conditions:

(1) $\zeta_{n}$ is disjoint; 
(2) $\zeta_{n}$ consists of coinciding one-point sets.

According to (3), we have $\bigcap_{n \in \omega} u\left(\left(U_{n, k}\right)_{k \in \omega}\right) \neq \varnothing$. By virtue of Statement 9 the sequence $\left(W_{k}\right)_{k \in \omega}$ is not locally finite.

The equivalence $(3) \Longleftrightarrow(4)$ was proved in [11].

Definition 2. Let $p \in \omega^{*}$ be an ultrafilter. We say that a space $X$ is discretely $p$-compact if any discrete exact sequence $\left(x_{n}\right)_{n \in \omega} \subset X$ has a $p$-limit.

Definition 3. Let $p \in \omega^{*}$ be an ultrafilter. We say that a space $X$ is sequentially $p$-compact if any infinite set $M \subset X$ has an infinite subset $L \subset M$ such that any exact sequence $\left(x_{n}\right)_{n \in \omega} \subset L$ has a $p$-limit.

Any infinite set contains an choose infinite discrete subset. This implies the following assertion.

Proposition 10. Let $p \in \omega^{*}$ be an ultrafilter. Then any discrete $p$-compact space is sequentially p-compact.

Proposition 11. Let $X$ be a sequentially p-compact space. Then $X^{\omega}$ is sequentially p-compact.

Proof. Take an infinite set $M \subset X^{\omega}$. By virtue of Statement 1 there exists an exact sequence $\left(x_{k}\right)_{k \in \omega} \subset M$ such that $\left(\pi_{n}\left(x_{k}\right)_{k \in \omega}\right.$ is an almost stationary or almost exact discrete sequence in $X$ for each $n$ (here $\pi_{n}: X \rightarrow X$ denotes the projection onto the $n$th factor). There exists a decreasing sequence $\left(S_{n}\right)_{n \in \omega}$ of infinite subsets of $\omega$ such that, for each $n \in \omega$, one of the following conditions holds:

(1) $\pi_{n}\left(x_{i}\right)=\pi_{n}\left(x_{j}\right)$ for $i, j \in S_{n}$;

(2) $\pi_{n}\left(x_{i}\right) \neq \pi_{n}\left(x_{j}\right)$ for any different $i, j \in S_{n}$ and each exact sequence $\left(y_{l}\right)_{l \in \omega} \subset\left\{\pi_{n}\left(x_{k}\right): k \in S_{n}\right\}$ has a $p$-limit.

Take an exact sequence $\left(s_{n}\right)_{n \in \omega}$ such that $s_{n} \in S_{n}$ for all $n$. Let $L=\left\{x_{s_{k}}\right.$ : $k \in \omega\}$. Then any exact sequence $\left(y_{n}\right)_{n \in \omega} \subset L$ has a $p$-limit.

Proposition 12. Let $X$ be a compact $\beta \omega$ space, and let $M \subset X,|M|<2^{2^{\omega}}$. Then $Y=X \backslash M$ is a discrete $p$-compact space for some $p \in \omega^{*}$.

Proof. Suppose that, on the contrary, there exist $\zeta_{p} \in \mathfrak{S}_{d}(Y)$ such that $\lim _{p} \zeta_{p} \notin$ $Y$ for all $p \in \omega^{*}$. By virtue of Statement [6, there exists a set $C \subset \omega^{*},|C|=2^{2^{\omega}}$, which consists of pairwise $\leq_{K R}$-incomparable ultrafilters. Since $|C|>|M|$, it follows that $x=\lim _{p} \zeta_{p}=\lim _{q} \zeta_{q} \in M$ for some different $p, q \in C$. We have $p, q \in \operatorname{sp}_{k}(x, X)$, which contradicts Proposition 3 . 


\section{Homogeneous Product Spaces}

Given a cardinal $\tau$, a set $A$, a family of spaces $\left(X_{\alpha}\right)_{\alpha \in A}$, and a point $\left(x_{\alpha}\right)_{\alpha \in A} \in$ $\prod_{\alpha \in A} X_{\alpha}$, we set We denote

$$
\begin{aligned}
& \sigma_{\tau}\left\{\left(X_{\alpha}, x_{\alpha}\right): \alpha \in A\right\}=\left\{\left(y_{\alpha}\right)_{\alpha \in A} \in \prod_{\alpha \in A} X_{\alpha}:\left|\left\{\alpha \in A: x_{\alpha} \neq y_{\alpha}\right\}\right|<\tau\right\}, \\
& \Sigma_{\tau}\left\{\left(X_{\alpha}, x_{\alpha}\right): \alpha \in A\right\}=\sigma_{\tau^{+}}\left\{\left(X_{\alpha}, x_{\alpha}\right): \alpha \in A\right\} .
\end{aligned}
$$

We also set

$$
H_{\tau}(X)=\sigma_{\tau}\{(X, x):(x, \alpha) \in X \times \tau\}
$$

for any space $X$. The space $H_{\omega_{1}}(X)$ is a $\Sigma$-product in $X^{X \times \omega_{1}}$, and $H_{\omega}(X)$ is a $\sigma$-product in $X^{X \times \omega}$.

Proposition $13(9])$. Let $X$ be a space, and let $\tau$ be an infinite cardinal. Then the space $H_{\tau}(X)$ is homogeneous and homeomorphic to $X \times H_{\tau}(X)$.

Proposition $14(9])$. Let $X$ be a space, and let $Y=H_{\omega_{1}}(X)$. If $X$ is $p$ compact for some $p \in \omega^{*}$, then so is $Y$.

Proposition 15. Let $X$ be a space, and let $Y=H_{\omega_{1}}(X)$. If $X$ is sequentially $p$-compact for some $p \in \omega^{*}$, then so is $Y$.

Proof. Let $M \subset Y,|M|=\omega$. There exists a closed set $F \subset Y$ such that $M \subset F$ and $F$ is homeomorphic to $X^{\omega}$. Proposition 11 implies the sequential $p$-compactness of $F$. Hence there exists an infinite set $L \subset M$ such that any exact sequence $\left(x_{n}\right)_{n \in \omega} \subset L$ has a $p$-limit in $F \subset Y$.

Proposition 16. For any $p \in \omega^{*}$, there exist extremally disconnected spaces $X$ and $Y$ such that $X$ is p-compact, $Y$ is sequentially q-compact for some $q \in \omega^{*}$, and $X \times Y$ is not pseudocompact.

Proof. Let $X$ be a minimal $p$-compact subspace of $\beta \omega$ containing $\omega$. Then $|X| \leq 2^{\omega}$. We set $Y=\omega \cup(\beta \omega \backslash X)$. Since $X \cap Y=\omega$, it follows that $X \times Y$ is not pseudocompact, and since $|\beta \omega \backslash Y| \leq|X| \leq 2^{\omega}$, it follows by Proposition 12 that $Y$ is sequentially $q$-compact for some $q \in \omega^{*}$.

Theorem 1. For any $p \in \omega^{*}$, there exist homogeneous spaces $X$ and $Y$ such that $X$ is p-compact, $Y$ is sequentially $q$-compact for some $q \in \omega^{*}$, and $X \times Y$ is not pseudocompact. Moreover, the space $X^{\tau}$ is countably compact for any $\tau$ and $Y^{\omega}$ is countably compact.

Proof. By virtue of Proposition [16, there exist extremally disconnected spaces $X^{\prime}$ and $Y^{\prime}$ such that $X^{\prime}$ is $p$-compact, $Y^{\prime}$ is sequentially $q$-compact for some $q \in$ $\omega^{*}$, and $X^{\prime} \times Y^{\prime}$ is not pseudocompact. We set $X=H_{\omega_{1}}\left(X^{\prime}\right)$ and $Y=H_{\omega_{1}}\left(Y^{\prime}\right)$. According to Proposition 13, the spaces $X$ and $Y$ are homogeneous and $X^{\prime} \times Y^{\prime}$ is a continuous image of $X \times Y$. Therefore, $X \times Y$ is not pseudocompact. By Propositions 14 and 15 the space $X$ is $p$-compact and $Y$ is sequentially $q$ compact, and by Proposition 11 the product space $Y^{\omega}$ is countably compact. 


\section{Maximally Homogeneous Spaces}

Given a space $X$, we denote the group of self-homeomorphisms of $X$ by Aut(X) and set

$$
H(X)=\{g(x): x \in X, g \in \operatorname{Aut}(\beta \mathrm{X})\} .
$$

The following assertion is a direct consequence of these definitions.

Statement 10. If $X$ is a space, then $H(H(X))=H(X)$. If $X$ is a homogeneous space, then so is $H(X)$.

Definition 4. We say that a space $X$ is maximally homogeneous if $X$ is homogeneous and $H(X)=X$.

This definition immediately implies the following assertion.

Statement 11. If $X$ is a homogeneous space, then $H(X)$ is maximally homogeneous.

Statement 12. If $X$ is an extremally disconnected space, $U, V \subset X$ are nonempty disjoint open subsets of $X$, and $f: U \rightarrow V$ is a homeomorphism, then there exists an $\tilde{f} \in \operatorname{Aut}(\beta \mathrm{X})$ for which $\tilde{f} \uparrow_{U}=f, \tilde{f} \uparrow_{V}=f^{-1}$, and $\tilde{f} \uparrow_{S}=\operatorname{id}_{S}$, where $S=\beta X \backslash(\bar{U} \cap \bar{V})$.

Proof. We set $W=U \cup V$. Consider the homeomorphism $g: W \rightarrow W$ defined by

$$
g(x)= \begin{cases}f(x) & \text { if } x \in U, \\ f^{-1}(x) & \text { if } x \in V .\end{cases}
$$

Let $\tilde{g}: \beta W \rightarrow \beta W$ be the continuous extension of $g$, and let $\tilde{f}: \beta X \rightarrow \beta X$ be the homeomorphism defined by

$$
\tilde{f}(x)= \begin{cases}\tilde{g}(x) & \text { if } x \in \beta W \\ x & \text { if } x \in \beta X \backslash \beta W .\end{cases}
$$

Statement 12 has the following corollary.

Corollary 1. If $X$ is a maximally homogeneous extremally disconnected space, $U, V \subset X$ are nonempty disjoint open subsets of $X$, and $f: U \rightarrow V$ is a homeomorphism, then there exists an $\tilde{f} \in \operatorname{Aut}(\mathrm{X})$ for which $\tilde{f} \uparrow_{U}=f, \tilde{f} \uparrow_{V}=f^{-1}$, and $\tilde{f} \uparrow_{S}=\operatorname{id}_{S}$, where $S=X \backslash(\bar{U} \cap \bar{V})$.

Statement 13. Let $X$ be a maximally homogeneous nondiscrete extremally disconnected space, and let $\left(x_{n}\right)_{n \in \omega},\left(y_{n}\right)_{n \in \omega} \in \mathfrak{S}_{d}(X)$. Then there exists an $f \in \operatorname{Aut}(\mathrm{X})$ such that $f\left(x_{n}\right)=y_{n}$ for all $n \in \omega$. 
Proof. There exists a nonempty clopen set $O \subset X$ such that

$$
O \cap \overline{\left(x_{n}\right)_{n \in \omega}}=O \cap \overline{\left(y_{n}\right)_{n \in \omega}}=\varnothing .
$$

Take $\left(z_{n}\right)_{n \in \omega} \in \mathfrak{S}_{d}(X)$ for which $\left(z_{n}\right)_{n \in \omega} \subset O$.

Let us show that there exists a $g \in \operatorname{Aut}(\mathrm{X})$ such that $g\left(x_{n}\right)=z_{n}$ for all $n \in \omega$. For each $n \in \omega$, take $g_{n} \in \operatorname{Aut}(\mathrm{X})$ taking $x_{n}$ to $z_{n}$. There exist sequences $\left(U_{n}\right)_{n \in \omega},\left(V_{n}\right)_{n \in \omega} \in \mathfrak{O}_{d}(X)$ satisfying the conditions $g_{n}\left(U_{n}\right)=V_{n}$, $x_{n} \in U_{n} \subset X \backslash W$, and $z_{n} \in V_{n} \subset W$ for each $n \in \omega$. We set $U=\bigcup_{n \in \omega} U_{n}$ and $V=\bigcup_{V_{n} \in \omega} U_{n}$ and consider the homeomorphism $g^{\prime}: U \rightarrow V$ defined by $g^{\prime}(x)=g_{n}(x)$ if $x \in U_{n}, n \in \omega$. Corollary 1 implies the existence of a $g \in \operatorname{Aut}(\mathrm{X})$ for which $g \uparrow_{U}=g^{\prime}, g \uparrow_{V}=g^{\prime-1}$, and $g\left\lceil_{S}=\operatorname{id}_{S}\right.$, where $S=X \backslash(\bar{U} \cap \bar{V})$.

Similarly, there exists an $h \in \operatorname{Aut}(\mathrm{X})$ such that $h\left(y_{n}\right)=z_{n}$ for all $n \in \omega$. It remains to set $f=h^{-1} \circ g$.

Corollary 2. Let $X$ be a maximally homogeneous extremally disconnected space. Then $u(\zeta)=u(\rho)$ for all $\zeta, \rho \in \mathfrak{S}_{d}(X)$.

Proposition 17. Let $X$ be a maximally homogeneous extremally disconnected space containing a countable nonclosed discrete subspace. Then $X$ is discretely p-compact for some free ultrafilter $p \in \omega^{*}$.

Proof. Let $\zeta \in \mathfrak{S}_{d}(X)$ be a nonclosed discrete sequence. Then $u(\zeta) \neq \varnothing$. Take $p \in u(\zeta)$. Let us show that $X$ is discretely $p$-compact. If $\rho \in \mathfrak{S}_{d}(X)$, then $u(\rho)=u(\zeta) \ni p$ by Corollary 2 . Therefore, $\rho$ has a $p$-limit in $X$.

Propositions 17 and 11 imply the following assertion.

Corollary 3. Let $X$ be a countably compact maximally homogeneous extremally disconnected space. Then $X^{\omega}$ is countably compact.

Lemma 1. Let $X$ be an extremally disconnected space which is not a $P$-space. Then there exists a point $x_{*} \in X$ and a sequence $\left(O_{n}\right)_{n \in \omega} \mathfrak{O}(X)$ such that $O_{n}$ is clopen and $x_{*} \in O_{n+1} \subset O_{n}$ for each $n \in \omega$ and the set $\bigcap_{n \in \omega} O_{n}$ is nowhere dense in $X$.

Proof. There exists a point $x_{*} \in X$ and a sequence $\left(U_{n}\right)_{n \in \omega} \mathfrak{O}(X)$ such that $x_{*} \in U_{n+1} \subset$ subset $O_{n}$ and $U_{n}$ is clopen for each $n \in \omega$ and $x_{*} \notin \operatorname{int}(F)$, where $F=\bigcap_{n \in \omega} O_{n}$. The set $F$ is closed, and since $X$ is extremally disconnected, it follows that $\operatorname{int}(F)$ is clopen. It remains to set $O_{n}=U_{n} \backslash \operatorname{int}(F)$.

Proposition 18. Let $X$ be a homogeneous extremally disconnected space which is not a P-space. Then $X^{\omega}$ is pseudocompact.

Proof. Using Lemma 1, we take $x_{*} \in X$ and $\left(O_{n}\right)_{n \in \omega} \mathfrak{O}(X)$ such that $O_{n}$ is clopen and $x_{*} \in O_{n+1} \subset O_{n}$ for each $n \in \omega$ and the set $\bigcap_{n \in \omega} O_{n}$ is nowhere dense in $X$.

Let $\zeta_{n}=\left(U_{n, k}\right)_{k \in \omega} \in \mathfrak{O}_{n}(X)$. By virtue of Proposition 9, to prove the proposition, it suffices to show that $\bigcap_{n \in \omega} u\left(\zeta_{n}\right) \neq \varnothing$. 
Since $X$ is a zero-dimensional homogeneous space, it follows that, for each $k \in \omega$, there exist homeomorphic clopen sets $V_{k}, V_{0, k}, V_{1, k}, \ldots, V_{k, k}$ such that $x_{*} \in V_{k} \subset O_{k}, S_{k}=V_{k} \backslash V_{k+1} \neq \varnothing$, and $V_{n, k} \subset U_{n, k}$ for $n=0,1, \ldots, k$. Let $h_{n, k}: V_{k} \rightarrow V_{n, k}$ be homeomorphisms, and let $S_{n, k}=h_{n, k}\left(S_{k}\right)$ for $n=$ $0,1, \ldots, k$. We set

$$
W_{n, k}= \begin{cases}U_{n, k}, & k<n, \\ S_{n, k}, & k \geq n\end{cases}
$$

for $n, k \in \omega$.

Lemma 2. The following assertions hold:

(1) $u\left(\left(S_{k}\right)_{k \in \omega}\right) \neq \varnothing$;

(2) $u\left(\left(S_{k}\right)_{k \in \omega}\right)=u\left(\left(W_{n, k}\right)_{k \in \omega}\right)$ for any $n \in \omega$.

Proof. We set $F=\bigcap_{k \in \omega} V_{k}$ and $Q_{k}=\bigcup_{j \geq k} S_{j}$. By construction we have $Q_{k}=V_{k} \backslash F$.

(1) Since $P=\bigcap_{n \in \omega} O_{n}$ is nowhere dense in $X$ and $F \subset P$, it follows that $F$ is nowhere dense in $X$. Therefore, $\overline{Q_{0}}=\overline{V_{0} \backslash F}=V_{0}$. We have $x_{*} \in \overline{Q_{0}} \backslash Q_{0}$, because $x_{*} \in F$. Hence $x_{*}$ is an accumulation point for the sequence $\left(S_{k}\right)_{k \in \omega}$ and $u\left(\left(S_{k}\right)_{k \in \omega}\right) \neq \varnothing$.

(2) We set $P_{n}=\bigcup_{k>n} S_{n, k}$. Consider the homeomorphism $f_{n}: Q_{n} \rightarrow P_{n}$ defined by $f_{n}(x)=h_{n, k}(x)$ if $x \in S_{k}$. Corollary 1 implies the existence of a $\tilde{f}_{n} \in \operatorname{Aut}(\mathrm{X})$, for which that $\tilde{f}_{n} \Upsilon_{Q_{n}}=f_{n}$. Since $\tilde{f}_{n}\left(S_{k}\right)=S_{n, k}=W_{n, k}$ for $k \geq n$, we have $u\left(\left(S_{k}\right)_{k \in \omega}\right)=u\left(\left(W_{n, k}\right)_{k \in \omega}\right)$.

The inclusions $W_{n, k} \subset U_{n, k}$ for $n, k \in \omega$ and Lemma 2 imply

$$
\bigcap_{n \in \omega} u\left(\zeta_{n}\right) \supset \bigcap_{n \in \omega} u\left(\left(W_{n, k}\right)_{k \in \omega}\right)=u\left(\left(S_{k}\right)_{k \in \omega}\right) \neq \varnothing .
$$

\section{Homogeneous Subspaces of Product Spaces}

Theorem 2. Let $X$ be a homogeneous $\beta \omega$ space. Then any compact subspace of $X$ is finite.

Proof. Suppose that, on the contrary, $X$ contains an infinite compact subspace. By Proposition 1 we have $\operatorname{sp}_{k}(x, X)=\omega^{*}$ for each $x \in X$, which contradicts Propositions 3 and 6 .

Corollary 4. Any compact subspace of a homogeneous extremally disconnected space is finite.

Corollary 4 is also implied by Theorem 2(c) in 22 . 
Theorem 3. (CH) Let $Y$ be a $\beta \omega$ space, and let $X \subset Y^{\omega}$ be a homogeneous space. Then any compact subspace of $X$ metrizable.

Proof. Suppose that, on the contrary, $X$ contains a nonmetrizable compact subspace $K$. Then Statement 7 implies the existence of a sequence $\zeta=\left(z_{m}\right)_{m \in \omega} \in$ $\mathfrak{S}_{d k}(X)$ such that $\bar{\zeta}$ is homeomorphic to $\beta \omega$. Take $z \in X$. For each $p \in \omega^{*}$, we fix $f_{p} \in \operatorname{Aut}(\mathrm{X})$ such that $f_{p}\left(\lim _{p} \zeta\right)=z$.

By virtue of Proposition 7 there exists a $C \subset \omega^{*},|C|=2^{2^{\omega}}$, consisting of pairwise $\leq_{K R}$-incomparable selective ultrafilters.

Statement 8 implies that, for each $p \in C$, there exists an $m_{p} \in \omega$ and an $N_{p} \in p$ such that the set $\left\{\pi_{m_{p}}\left(z_{n}\right): n \in N_{p}\right\}$ is discrete and $\pi_{m_{p}}\left(x_{j}\right) \neq$ $\pi_{m_{p}}\left(x_{i}\right)$ for any different $i, j \in N_{p}$. Since $|C|>\omega$, there are different $p, q \in C$ for which $m=m_{p}=m_{q}$. Therefore, $p, q \in \operatorname{sp}_{k}\left(\pi_{m}(z), X\right)$. This contradicts Proposition 3 .

Corollary 5. $(\mathrm{CH})$ Let $Y$ be a $\beta \omega$ space, and let $X \subset Y^{\omega}$ be a homogeneous compact space. Then $X$ is metrizable.

Theorem 4. $(\mathrm{CH})$ Let $Y$ be a $\beta \omega$ space, and let $X$ be a homogeneous subspace of $Y^{n}$ for some $n \in \omega$. Then any compact subspace of $X$ is finite.

Proof. Let $K \subset X$ be a compact set. Then $K$ is metrizable by Theorem 3 , Since all metrizable compact sets in any $\beta \omega$ space are finite, it follows that the projection of $K$ on any factor in $Y^{n}$ is finite. Therefore, $K$ is finite.

Question 1. Are Theorem 3 and 4 true naïvely? What if $Y$ is additionally assumed to be an F-space (an ED space)?

In what follows, we treat positive integers as sets of smaller integers.

Let $X \subset Y^{n}$, where $X$ and $Y$ are sets and $k, n \in \omega$. We write $\operatorname{dim}_{p}(X) \geq k$ if there exists a set $M \subset n,|M|=k$, and a sequence $\left(x_{i}\right)_{i \in \omega} \subset X$ such that $\pi_{m}\left(x_{i}\right) \neq \pi_{m}\left(x_{j}\right)$ for any $m \in M$ and any different $i, j \in \omega$. We set

$$
\operatorname{dim}_{p}(X)=\max \left\{m: \operatorname{dim}_{p}(X) \geq m\right\} .
$$

Let $\mathcal{P}(Y, n, k)$ denote the family of sets of the form $\{p\} \times Y^{M}$, where $M \subset n$, $|M|=k$, and $p \in Y^{n \backslash M}$. We set

$$
\mathcal{P}^{*}(Y, n, k)=\{\bigcup \gamma: \gamma \subset \mathcal{P}(Y, n, k),|\gamma|<\omega\} .
$$

Given $q \in Y^{n}$, we put

$$
\begin{aligned}
& \mathcal{P}_{q}(Y, n, k)=\left\{\left\{q \uparrow_{n \backslash M}\right\} \times Y^{M}: M \subset n,|M|=k\right\}, \\
& \mathcal{P}_{q}^{*}(Y, n, k)=\bigcup \mathcal{P}_{q}(Y, n, k) .
\end{aligned}
$$

Statement 14. Suppose that $Y$ is an infinite set, $n \in \omega, k \leq n$, and $X \subset Y^{n}$. Then the following assertions hold:

(1) if $Z \subset X$, then $\operatorname{dim}_{p}(Z) \leq \operatorname{dim}_{p}(X)$; 
(2) if $X_{1}, X_{2} \subset Y^{n}$ and $X=X_{1} \cap X_{2}$, then

$$
\operatorname{dim}_{p}(X)=\max \left(\operatorname{dim}_{p}\left(X_{1}\right), \operatorname{dim}_{p}\left(X_{2}\right)\right)
$$

(3) $\operatorname{dim}_{p}(Z)=k$ for all $Z \in \mathcal{P}^{*}(Y, n, k)$;

(4) given $q, r \in Y^{n}, q \in \mathcal{P}_{r}(Y, n, k)$ if and only if $\left|\left\{i<n: \pi_{i}(q) \neq \pi_{i}(r)\right\}\right| \leq$ $k$;

(5) $\operatorname{dim}_{p}(X) \leq k$ if and only if $X \subset M$ for some $M \in \mathcal{P}^{*}(Y, n, k)$;

(6) if $Y$ is a space, $q \in X$, and $\operatorname{dim}_{p}(X) \leq k$, then $q \in \operatorname{int}_{X}\left(X \cap \mathcal{P}_{q}^{*}(Y, n, k)\right)$.

Proof. Assertion (1) is obvious.

Assertion (2) follows from the observation that if $\operatorname{dim}_{p}(X) \geq k$, then, by definition, we have either $\operatorname{dim}_{p}\left(X_{1}\right) \geq k$ or $\operatorname{dim}_{p}\left(X_{2}\right) \geq k$.

Let us prove (3). Obviously, we have $\operatorname{dim}_{p}(Q)=k$ for $Q \in \mathcal{P}(Y, n, k)$, so that it suffices to apply (2).

Assertion (4) follows from the definitions.

Let us prove (5). Assertions (1) and (3) imply that if $X \subset M$ for some $M \in \mathcal{P}^{*}(Y, n, k)$, then $\operatorname{dim}_{p}(X) \leq k$. Let us prove that if $\operatorname{dim}_{p}(X) \leq k$, then $X \subset M$ for some $M \in \mathcal{P}^{*}(Y, n, k)$. Assume the contrary. Let us construct a sequence $\left(q_{n}\right)_{n \in \omega} \subset X$ by induction on $n$ as follows. Take any $q_{0} \in X$. Having constructed $q_{0}, \ldots, q_{n-1} \in X$, we choose $q_{n} \in X \backslash \bigcup_{i=0}^{n-1} \mathcal{P}_{q_{i}}^{*}(Y, n, k)$. Note that $q_{m} \notin \mathcal{P}_{q_{n}}^{*}(Y, n, k)$ for $m, n \in \omega$. Consider the set $M_{n, m}=\{i<n$ : $\left.\pi_{i}\left(q_{n}\right) \neq \pi_{m}\right\}$; according to assertion (4), we have $\left|M_{n, m}\right|>k$. Let $\mathcal{M}$ denote the family of all finite sets $n$. We color the complete graph on the $\omega$ elements of $\mathcal{M}$ by assigning the set $M_{n, m}$ to each edge $\{n, m\}$. According to Ramsey's theorem, there exists an infinite set $M \subset \omega$ such that $M=M_{n, m}=M_{n^{\prime}, m^{\prime}}$ for $n, m, n^{\prime} m^{\prime} \in \omega, n \neq m, n^{\prime} \neq m^{\prime}$. By definition, we have $\operatorname{dim}_{p}(X) \geq|M|>k$.

Let us prove (6). Assertion (5) implies the existence of a finite set $\gamma \subset$ $\mathcal{P}(Y, n, k)$ for which $X \subset \bigcap \gamma$. Since $\gamma$ consists of closed sets, it follows that $x \in \operatorname{int}_{X}\left(X \cap \bigcap \gamma_{1}\right)$ and $x \notin \overline{X \cap \bigcap \gamma_{1}}$, where $\gamma_{1}=\{F \in \gamma: q \in F\}$ and $\gamma_{1}=\{F \in \gamma: q \notin F\}$. The inclusion $\gamma_{1} \subset \mathcal{P}_{q}(Y, n, k)$ implies $q \in \operatorname{int}_{X}(X \cap$ $\left.\mathcal{P}_{q}^{*}(Y, n, k)\right)$.

Proposition 19. Suppose that $Y$ is an F-space, $n \in \omega, n>1$, and $X \subset Y^{n}$ is a homogeneous space. Then $\operatorname{dim}_{p}(K)<n-1$ for any compact subspace $K \subset X$.

Proof. Assume the contrary. Then $\operatorname{dim}_{p}(K) \geq n-1$. There exists a set $M \subset n$ with $|M|=n-1$ and a sequence $\zeta=\left(x_{i}\right)_{i \in \omega} \subset K$ such that $\pi_{m}\left(x_{i}\right) \neq \pi_{m}\left(x_{j}\right)$ for $m \in M$. We can assume without loss of generality that $M=\{1,2, \ldots, n-1\}$. Passing to a subsequences if necessary, we can also assume that the sequences $\zeta_{i}=\left(\pi_{i}\left(x_{j}\right)\right)_{j \in \omega}$ are discrete and $\zeta_{i} \in \mathfrak{S}_{d k}(X)$ for $i>0$. Proposition 6 implies the existence of three pairwise $\leq_{K R}$-incomparable weak $P$ ultrafilters $p, q$, and $r$. Let $x=\lim _{p} \zeta=\left(x_{0}, \ldots, x_{n-1}\right)$. By virtue of Proposition 1 we have $\operatorname{sp}_{k}(x, X)=$ $\omega^{*}$. There exist $\xi=\left(u_{n}\right)_{n \in \omega}$ and $\rho=\left(v_{n}\right)_{n \in \omega} \in \mathfrak{S}_{d k}(X)$ for which $x=$ $\lim _{q} \xi=\lim _{r} \rho$. According to Proposition [5] for $i=1,2, \ldots, n-1$, we have 
$M_{i}=\left\{n \in \omega: \pi_{i}\left(u_{n}\right)=x_{i}\right\} \in q$ and $N_{i}=\left\{n \in \omega: \pi_{i}\left(v_{n}\right)=x_{i}\right\} \in r$. We set $M=\bigcap_{i=1}^{n-1} M_{i}$ and $N=\bigcap_{i=1}^{n-1} N_{i}$. Then $M \in q, N \in r,\left\{u_{n}: \in M\right\} \subset F$, and $\left\{v_{n}: \in N\right\} \subset F$, where $F=\left\{\left(w, x_{1}, \ldots, x_{n-1}: w \in Y\right\}\right.$. The space $F$ is homeomorphic to $Y$, and $q, r \in \operatorname{sp}_{k}(x, F)$. This contradicts Proposition 3 ,

Proposition 20. If $Y$ is a $\beta \omega$ space, $n \in \omega$, and $X \subset Y^{n}$ is homogeneous, then $\operatorname{dim}_{p}(K)>1$ for any infinite compact space $K \subset X$.

Proof. Suppose that, on the contrary, $X$ contains an infinite compact set and $\operatorname{dim}_{p}(K) \leq 1$ for any compact space $K \subset X$. Let $q \in X$. By Proposition 1 we have $\operatorname{sp}_{k}(q, X)=\omega^{*}$. Proposition 6 implies the existence of $n+1$ pairwise incomparable ultrafilters $r_{0}, r_{1}, \ldots, r_{n} \in \omega^{*}$. For each $i<n+1$, choose $\zeta_{i}=$ $\left(x_{i, k}\right)_{k \in \in \omega} \in \mathfrak{S}_{d k}(X)$ so that $q=\lim _{r_{i}} \zeta_{i}$. For $K=\bigcup_{i=0}^{n} \bar{\zeta}_{i}$, we have $\operatorname{dim}_{p} K=$ 1. Let $\mathcal{P}_{q}(X, n, 1)=\left\{F_{0}, F_{1}, \ldots, F_{n-1}\right\}$. It follows from Statement 14 (6) that if $i \leq n$, then $\left\{k \in \omega: x_{i, k} \in F_{m_{i}}\right\} \in \zeta_{i}$ for some $m_{i}<n$. Thus, $m=m_{i}=m_{j}$ for some $i<j<n$, so that $r_{i}, r_{j} \in \operatorname{sp}_{k}\left(q, F_{m}\right)$. Since $F_{m}$ is homeomorphic to $Y$ and $Y$ is a $\beta \omega$ space, we have obtained a contradiction to Proposition 3 .

Theorem 5. Let $Y$ F-space, and let $X \subset Y^{3}$ be a homogeneous space. Then any compact subspace of $X$ is finite.

Proof. Suppose that, on the contrary, $X$ contains an infinite compact set $K$. Then Proposition 19 implies $\operatorname{dim}_{p} K<3-1=2$, and Proposition 20 implies $\operatorname{dim}_{p} K>1$.

Question 2. Let $Y$ be an F-space, and let $X \subset Y^{4}$ be a homogeneous space. Is it true that any compact subspace of $X$ is finite?

Proposition 21. Suppose that $Y$ is a space, $n \in \omega, X \subset Y^{n}$ is a zerodimensional homogeneous compact space, and $k=\operatorname{dim}_{p} X$. Then there exists a finite space $Z$ such that $X$ is embedded in $Z \times Y^{k}$ and $(Z \times Y)^{k}$.

Proof. According to Statement 14(5), we have $X \subset \cup \gamma$ for some finite set $\gamma \subset \mathcal{P}(Y, n, k)$. The family $\mathcal{P}(Y, n, k)$ consists of closed subsets; therefore, $\operatorname{int}_{X}(X \cap F) \neq \varnothing$ for some $F \in \gamma$.

Since $X$ is a homogeneous zero-dimensional compact set, it follows that there exists a finite partition $\lambda$ of $X$ into clopen subsets such that each $U \in \lambda$ is embedded in $\operatorname{int}_{X}(X \cap F) \subset F$. The space $F$ is homeomorphic to $Y^{k}$; hence $X$ is embedded in $Z \times Y^{k}$, where $Z$ is a finite discrete space of cardinality $|Z|=|\gamma|$.

Theorem 6. If $Y$ is an F-space, $n \in \omega$, and $X \subset Y^{n}$ is a homogeneous compact space, then $X$ is finite.

Proof. Suppose that, on the contrary, $X$ is infinite. We can assume that $n$ is the least positive integer $m$ such that $X$ is embedded in the $m$ th power of an F-space. All F-spaces are zero-dimensional; therefore, $\operatorname{dim}_{p} X=n$ by Proposition 21. This contradicts Proposition 19. 


\section{References}

[1] W. W. Comfort and Kenneth A. Ross, Pseudocompactness and uniform continuity in topological groups, Pacific J. Math. 16, 483-496 (1966).

[2] W. Comfort and Jan van Mill, On the product of homogeneous spaces. Topol. Appl. 21, 297-308 (1985).

[3] W. W. Comfort and Jan van Mill, A homogeneous extremally disconnected countably compact space, Topol. Appl. 25 (1), 65-73 (1987).

[4] Akio Kato, A new construction of extremally disconnected topologies, Topol. Appl. 58 (1), 1-16 (1994).

[5] W. Lindgren and A. Szymanski, A Non-Pseudocompact Product of Countably Compact Spaces Via Seq, Proc. Amer. Math. Soc. 125 (12), 3741-3746 (1997).

[6] Zdeněk Frolík, Homogeneity problems for extremally disconnected spaces, Comment. Math. Univ. Carolinae 008.4, 757-763 (1967).

[7] Alexander Arhangel'skii and Mikhail Tkachenko, Topological Groups and Related Structures (Atlantis Press/World Sci., Paris, 2008).

[8] A. V. Arhangel'skii, Groupes topologiques extremalement discontinus, C. R. Acad. Sci. Paris 265, 822-825 (1967).

[9] E. A. Reznichenko, Homogeneous products of spaces, Mosc. Univ. Math. Bull. 51 (3), 6-8 (1996).

[10] A. Arhangel'skiǔ, Every extremally disconnected bicompactum is inhomogeneous, Soviet Math. Dokl. 8, 897-900 (1967).

[11] I. Glicksberg, Stone-Čech Compactifications of Products, in: The Mathematical Legacy of Eduard Čech, ed. by M. Katětov and P. Simon (Birkhäuser, Basel, 1993).

[12] L. Gillman and M. Jerison, Rings of Continuous Functions (Van Nostrand Reinhold, New York, 1960).

[13] B. Balcar and A. Dow, Dynamical systems on compact extremally disconnected spaces, Topology Appl. 41, 41-56 (1991).

[14] W. W. Comfort and S. Negrepontis, The Theory of Ultrafilters (Springer, Berlin, 1974).

[15] E. K. van Douwen, Prime mappings, number of factors and binary operations, Dissertationes Mathematicae 199 (1981).

[16] K. Kunen, Large homogeneous compact spaces, in: Open Problems in Topology (North-Holland, Amsterdam, 1990), pp. 261-270. 
[17] Saharon Shelah and M. E. Rudin, Unordered types of ultrafilters, Topol. Proc. 3, 199-204 (1979).

[18] K. Kunen, Weak $P$-points in $N^{*}$, Coll. Math. Soc. János Bolyai 23, 741-749 (1978).

[19] P. Simon, Applications of independent linked families, Colloq. Math. Soc. János Bolyai, 41, 561-580 (1985).

[20] W. Rudin, Homogeneity problems in the theory of Cech compactifications, Duke Math. J. 23, 409-420 (1956).

[21] M. E. Rudin, Partial orders on the types in $\beta N$, Trans. Amer. Math. Soc. 155 (2), 353-362 (1971).

[22] Eric K. van Douwen, Homogeneity of $\beta G$ if $G$ is a topological group, Colloq. Math. 41, 193-199 (1979).

[23] F. P. Ramsey, On a problem of formal logic, Proc. London Math. Soc. 30, 264-286 (1930). 